\title{
Psychosocial distress and functioning of Greek youth with cystic fibrosis: a cross-sectional study
}

\author{
Konstantina Kostakou', George Giannakopoulos ${ }^{1 *}$, Stavroula Diareme ${ }^{1}$, Chara Tzavara', Stavros Doudounakis², \\ Stelios Christogiorgos ${ }^{1}$, Chryssa Bakoula ${ }^{3}$ and Gerasimos Kolaitis ${ }^{1}$
}

\begin{abstract}
Background: To assess psychosocial functioning and distress of children and adolescents with cystic fibrosis compared to healthy controls.

Methods: Thirty-six patients with cystic fibrosis aged 8-18 years (24 boys, mean age \pm SD: $11.5 \pm 2.6$ years) and 31 sex- and age-matched healthy control subjects (18 boys, mean age \pm SD: $12 \pm 2.5$ years) were enrolled in the study. In order to assess the self-esteem, social adjustment, and family functioning of these young people, the Culture-free Self-esteem Inventory, the Social Adjustment Scale-Self-Report, and the Family Assessment Device were administered. Emotional/ behavioral problems were assessed through the Youth Self Report and the Child Behavior Checklist given to both the subjects and their parents.
\end{abstract}

Results: No significant differences were found for self-esteem between the two study groups. Regarding social adjustment, children with cystic fibrosis reported significantly worse friendship and overall adjustment $(P<0.05)$. Moreover, no difference was found in the levels of family functioning between the two groups. No significant differences between the groups were found in emotional/ behavioral problems from the self-reports. On the contrary, parents of children with cystic fibrosis reported significantly higher levels of withdrawal/ depression, thought problems, and delinquent behavior $(P \leq 0.01)$ as compared to controls.

Conclusions: Children and adolescents with cystic fibrosis appear to be a psychosocially vulnerable group. A biopsychosocial approach should emphasize the assessment and treatment of the psychosocial distress of these patients alongside multiple somatic treatments.

Keywords: Children and adolescents, Cystic fibrosis, Family functioning, Psychopathology, Self-esteem, Social adjustment

\section{Background}

There is evidence that individuals with chronic medical conditions are at increased risk of psychiatric disorders and psychological distress [1-4]. Towards this end, there is a growing interest in psychosocial functioning among populations suffering from chronic diseases. Several studies have demonstrated that the risk for psychiatric problems in children with chronic medical conditions is about two to four times greater than that in their healthy peers $[2,5]$. Symptoms of anxiety and depression as well as behavioral problems appear to be more common in populations with

\footnotetext{
* Correspondence: giannakopoulos.med@gmail.com

'Department of Child Psychiatry, Athens University Medical School, Aghia

Sophia Children's Hospital, Athens, Greece

Full list of author information is available at the end of the article
}

chronic conditions than in the general population [6-8]. Additionally, the improvement in survival rates of most of the chronic conditions results in a growing need for further detection and evaluation of the psychological well-being of patients.

Cystic fibrosis (CF) is an example of a chronic illness with an improving survival rate. It is the most common life-limiting genetic disorder in white people, with a frequency of about 1 in 3500 live births [9,10], affecting almost 50.000 individuals in the European Union, with a mean prevalence of $0.737 / 10.000$ [11]. Life expectancy of CF patients is rising towards a mean of 40 years with advances in all aspects of therapy apart from treating the basic molecular defect $[12,13]$. CF is characterized by chronic airway obstruction and subsequent infection of 
the bronchial airways. Respiratory symptoms are experienced by nearly every patient. Gastrointestinal consequences include exocrine pancreatic insufficiency (in an estimated $90 \%$ of individuals), which sets the stage for CF-related diabetes in an estimated $50 \%$ of adults over the age of 30 years. Infertility is present for nearly $95 \%$ of males due to absence of the vas deferens and for $20 \%$ of females due to ion transport issues in the genitourinary system, such as abnormal cervical mucus [14]. Given the chronic, progressive and disabling nature of $\mathrm{CF}$, multiple treatments are required on a daily basis.

Developing a sense of self and acquiring autonomy in all areas of life are the major developmental tasks throughout childhood. However, normal developmental issues of puberty, autonomy, identity acquisition, sexuality and education may become more difficult tasks to deal with in CF patients $[15,16]$. Children's identity and sense of competence begin to be developed through a process of comparison with their peers [17]. Children with $\mathrm{CF}$ though have reduced growth velocity and delayed adolescent growth spurt [18], and such a comparison process may highlight their CF-related differences. Treatment burden, shortened mortality, and amplification of normal developmental issues may have an impact on the psychosocial well-being of young patients.

Consequently, there are numerous studies focusing on the psychosocial aspects of $\mathrm{CF}$, but the results have been variable. There is some evidence indicating that the psychological and psychosocial functioning of CF patients are similar to that of the healthy population [19-21]. However there is also evidence that pediatric patients do suffer an increased likelihood of psychiatric problems and psychological distress [22-24]. More specifically, although anxiety and depression appear to be more common in patients with CF than in the general population, symptoms of anxiety occur more frequently than depression in this population [25].

As far as the emotional needs of the young patients with CF are concerned, selection of informant appears to be an important variable that should be taken into consideration when those needs are being evaluated. More specifically, in parental ratings depressive symptoms [26] and behavior problems appear to be more frequent than in self ratings [6].

In Greece, there are almost 800 patients and the frequency of the disease is 1 in 3.500 live births, similar to that in the European Union [11]. The aim of the present study was the evaluation of the psychosocial functioning of Greek children and adolescents with CF. Family functioning, self-esteem, social adjustment, and emotional/ behavioral problems were examined and rates were compared to those of a group of healthy children and adolescents. The psychosocial functioning of children and adolescents with CF was expected to be worse than that of their healthy peers. Additionally, it was hypothesized that progression of age in the CF population might be followed by deterioration in emotional and behavioral functioning. Finally, parents and children were expected to show differences in their perceptions about the emotional/behavioral difficulties of these young patients.

\section{Methods}

\section{Participants and procedures}

Thirty-six patients with CF aged 8-18 years (24 boys, mean age \pm SD: $11.5 \pm 2.6$ years) and 31 sex- and agematched healthy control subjects (18 boys, mean age \pm SD: $12 \pm 2.5$ years) were enrolled in the study. All patients were followed-up as outpatients at regular intervals after the diagnosis at the CF Unit of Aghia Sophia Children's Hospital, Athens, Greece. The control subjects were recruited from a community sample and were healthy children without a sibling suffering from a chronic disease. The study was approved by the Scientific and Ethics Committees of Aghia Sophia Children's Hospital, and all parents provided written informed consent before entry of their children in the study.

\section{Measures}

All patients presented with good respiratory functioning (Forced Expiratory Volume- FEV\% 91.15 for girls/89.2 for boys), as assessed by spirometry on the day of the data collection. More specifically, 31 of our patients had mild pulmonary impairment (FEV\% predicted 70-100) and 5 of them had moderate pulmonary impairment (FEV\% predicted 40-70). None of our participants was assessed with a severe pulmonary impairment (FEV\% predicted <40).

Self-esteem was measured by the Culture-free Selfesteem Inventory (CFSEI) - Form B [27], a self-report scale that consists of 30 items measuring the individual's perception about the self. The items are divided into two groups: items that indicate high self-esteem and those that reveal low self-esteem. The respondent checks either yes or no for each item. The form has four scales: General Self-Esteem, Academic Self-Esteem, Parental/Home SelfEsteem, and Social Self-Esteem. The items can be summed to produce a Global Self-Esteem Quotient score, in addition to the subscale scores, with higher scores indicating higher self-esteem.

Social adjustment was measured by a modified version of the Social Adjustment Scale-Self-Report (SAS-SR; [28]). This version is appropriate for school-age children and early adolescents and assesses social functioning over the past two months $[29,30]$. It consists of 36 items categorized into four scales of nine items each: (a) School, (b) Friends, (c) Family, and (d) Overall Adjustment. Each item is scored on a 5-point scale, with higher scores indicating worse functioning. A sum-score for each scale is 
produced representing the mean rating of all items categorized in the respective scale.

Family functioning was assessed by the Family Assessment Device (FAD) [31], which was administered to the caregivers of the patients. The family assessment device is a 60-item, self-administered instrument which is used to assess six dimensions of family functioning - Problem Solving, Communication, Roles, Affective Responsiveness, Affective Involvement, and Behavior Control. Additionally, a General Functioning scale assesses overall functioning of the family. Answers in the 60 items are as follows: "I strongly agree", "I agree", "I disagree", "I strongly disagree". Subscale scores range from 1 to 4 . Lower scores suggest better functioning.

Patients' emotional/behavioral problems were measured through the Youth Self-report (YSR) and Child Behavior Checklist (CBCL) questionnaires [32]. The YSR is a selfreport questionnaire, administered to children and adolescents aged 11-18 years, while CBCL reflects parents' perception of their children's behavioral/emotional deficits. The CBCL is filled out by parents or surrogates of children aged 4 to 18 years. Both instruments contain 113 items rated on a three-point scale (i.e. $0=$ not true, $1=$ somewhat or sometimes true, $2=$ very true or often true), based on the preceding six months. The following eight syndromes are scored, Anxious/Depressed, Withdrawn/ Depressed, Somatic Complaints, Social Problems, Thought Problems, Attention Problems, Delinquent Behavior, and Aggressive Behavior. Withdrawn/Depressed, Somatic Complaints, and Anxious/Depressed syndromes comprise an Internalizing group, related to disturbances of emotion or behavioral deficits and the Aggressive Behavior and Delinquent Behavior syndromes comprise an Externalizing group, considered to reflect conduct disorders or behavioral excess.

\section{Data analysis}

Continuous variables are presented with mean \pm standard deviation while quantitative variables are presented with absolute and relative frequencies. For the comparisons of proportions chi-square tests were used. Student's t-tests were computed for the comparison of mean values. Differences in all study subscales according to age, sex, and the presence of CF were determined by the use of Multivariate Analysis of Variance (MANOVA). Logarithmic transformations were made for SAS-SR scales because they were not normally distributed. All $P$ values reported are two-tailed. Statistical significance was set at 0.05 and analyses were conducted using SPSS statistical software (version 13.0).

\section{Results}

The mean values for CFSEI, SAS-SR, and FAD scales for the control and CF group are described in Table 1. No significant differences were found for CFSEI scales between the two study groups. Furthermore, no gender or age differences were found for CFSEI scales defined by MANOVA in the CF and the control groups. Regarding SAS-SR dimensions, subjects with CF had significantly higher values $(P<0.05)$ for the Friends scale. Also significantly higher $(P<0.05)$ was the Overall Adjustment score of subjects with CF. Division of both patients and controls into two subgroups according to their age (8-11 and 12-18) revealed no differences in the SAS-SR scales. Also, division of both patients and controls in two subgroups according to the gender of the participant revealed no differences. Moreover, MANOVA indicated that there was no difference in the scores on FAD scales between the two groups. In the patient group (Table 2), parents of children aged 12-18 years $(\mathrm{n}=19)$ scored significantly higher $(P<0.05)$ on all FAD scales except for Behavior Control compared to younger children aged 8-11 years $(n=17)$. No such differences were found in the control group. No gender differences were found in either group.

In Table 3 the differences in the mean values of YSR and $\mathrm{CBCL}$ scales of the patient and control groups are presented. No significant differences were found between groups for self-reports. On the contrary, parents of subjects with CF reported significantly higher scores on Withdrawn/Depressed, Thought Problems, and Delinquent Behavior scales $(P \leq 0.01)$ as compared to controls. No gender or age effects were found.

\section{Discussion}

The aim of the present study was the evaluation of family functioning, self-esteem, social adjustment, and the presence of psychopathology among Greek children and adolescents with CF compared to a group of healthy children and adolescents. No significant differences were found between the two groups in family functioning. There is evidence that families with children and adolescents with CF show resilience and do not differ from families with healthy members as far as their functioning level is concerned [33-35]. On the contrary, further validation of the CF group after its division in two distinct subgroups based on age (8-11 and 12-18) demonstrated statistically significant differences in almost all of the dimensions. More specifically, families with younger children demonstrate better functioning.

As children enter into adolescence, family relations become more difficult. Adolescence is a period of rapid social, cognitive, and physiological changes. Peers become more influential and family time reduces relative to time spent with peers [17]. Persistent movement towards increasing autonomy, need for separation from parents and adolescent-parents relationships, as well as attempts to challenge parental authority may lead to greater family 
Table 1 Self-esteem, social adjustment, and family functioning of the patient and control groups

\begin{tabular}{|c|c|c|c|c|}
\hline \multirow{3}{*}{$\overline{\text { CFSEI }}$} & \multirow{2}{*}{$\begin{array}{l}\text { Controls } \\
\text { Mean } \pm \text { SD }\end{array}$} & \multicolumn{3}{|c|}{ Cystic fibrosis } \\
\hline & & \multirow[t]{2}{*}{ Mean \pm SD } & \multirow[t]{2}{*}{$F(\mathrm{df:1,} 63$ ) } & \multirow[t]{2}{*}{$P$ MANOVA* } \\
\hline & & & & \\
\hline General & $7.7 \pm 1.8$ & $8.3 \pm 1.4$ & 1.21 & 0.276 \\
\hline Academic & $4.1 \pm 1.2$ & $4.1 \pm 1.1$ & 0.00 & 0.949 \\
\hline Social & $3.5 \pm 0.8$ & $3.2 \pm 0.9$ & 1.84 & 0.181 \\
\hline Parental/Home & $4.5 \pm 0.8$ & $4.7 \pm 0.6$ & 0.38 & 0.540 \\
\hline Global quotient & $20 \pm 3$ & $20.6 \pm 2.8$ & 0.56 & 0.458 \\
\hline \multicolumn{5}{|l|}{ SAS-SR } \\
\hline Friends & $2.1 \pm 0.5$ & $2.4 \pm 0.5$ & 6.74 & 0.012 \\
\hline School & $1.7 \pm 0.4$ & $1.9 \pm 0.6$ & 3.78 & 0.056 \\
\hline Family & $1.8 \pm 0.4$ & $1.8 \pm 0.4$ & 0.38 & 0.847 \\
\hline Overall adjustment & $1.8 \pm 0.3$ & $2.0 \pm 0.4$ & 3.86 & 0.039 \\
\hline \multicolumn{5}{|l|}{ FAD } \\
\hline Problem solving & $1.7 \pm 0.3$ & $1.8 \pm 0.5$ & 0.43 & 0.514 \\
\hline Communication & $1.8 \pm 0.4$ & $1.9 \pm 0.5$ & 0.23 & 0.631 \\
\hline Roles & $2.2 \pm 0.4$ & $2.2 \pm 0.4$ & 0.04 & 0.841 \\
\hline Emotional responsiveness & $1.8 \pm 0.6$ & $1.8 \pm 0.6$ & 0.00 & 0.953 \\
\hline Emotional involvement & $1.9 \pm 0.4$ & $2.1 \pm 0.4$ & 2.24 & 0.140 \\
\hline Behavior control & $2 \pm 0.4$ & $2.1 \pm 0.5$ & 0.77 & 0.385 \\
\hline General functioning & $1.7 \pm 0.4$ & $1.7 \pm 0.4$ & 0.01 & 0.941 \\
\hline
\end{tabular}

CFSEI, Culture-free Self-esteem Inventory; SAS-SR, Social Adjustment Scale-Self-Report; FAD, Family Assessment Device.

*sex and age were used as covariates.

conflict, less parent-child cohesion, and poorer communication [36,37]. One could argue that this finding could primarily be interpreted as a result of normal and expected tension in family relations secondary to adolescence.

On the other hand, chronic conditions amplify normal family issues. Adolescents with CF, despite their maturation and their normal tendency and need for autonomy, are kept dependent on their parents and members of their medical team $[38,39]$. In this context the patients have to face developmental challenges related to puberty, identity acquisition, and sexuality $[15,16]$.

Additionally, age progression in CF is related to severity of illness and deterioration of health. CF typically worsens during adolescence and the presence of more frequent symptoms and pulmonary exacerbations correspond with greater illness burden [40]. Rising fears and fantasies may lead to emotional isolation and impede family communication.

No difference was found between the self-concept of healthy and CF children. Although there is evidence that chronic illness is a risk factor for low self-esteem [41], as far as patients with CF are concerned, there are previous relevant studies presenting results that are consistent with ours $[42,43]$. More specifically, research on the psychosocial characteristics of patients with chronic somatic conditions has revealed that adolescents with chronic

Table 2 Family functioning of the patient group according to sex and age

\begin{tabular}{|c|c|c|c|c|c|c|c|c|}
\hline FAD & $\begin{array}{c}\text { Boys } \\
\text { Mean } \pm \text { SD }\end{array}$ & $\begin{array}{c}\text { Girls } \\
\text { Mean } \pm \text { SD }\end{array}$ & F (df:1,33) & P MANOVA & $\begin{array}{l}\text { 8-11 years } \\
\text { Mean } \pm \text { SD }\end{array}$ & $\begin{array}{l}\text { 12-18 years } \\
\text { Mean } \pm \text { SD }\end{array}$ & F (df:1,33) & P MANOVA \\
\hline Problem solving & $1.7 \pm 0.5$ & $1.9 \pm 0.6$ & 0.01 & 0.939 & $1.5 \pm 0.4$ & $2 \pm 0.6$ & 4.88 & 0.035 \\
\hline Communication & $1.8 \pm 0.5$ & $1.9 \pm 0.5$ & 0.13 & 0.724 & $1.6 \pm 0.4$ & $2.1 \pm 0.4$ & 8.79 & 0.006 \\
\hline Roles & $2.2 \pm 0.4$ & $2.1 \pm 0.4$ & 1.51 & 0.230 & $2 \pm 0.3$ & $2.3 \pm 0.4$ & 5.41 & 0.027 \\
\hline Emotional responsiveness & $1.7 \pm 0.6$ & $1.9 \pm 0.6$ & 0.07 & 0.790 & $1.5 \pm 0.5$ & $2 \pm 0.6$ & 4.98 & 0.034 \\
\hline Emotional involvement & $2 \pm 0.3$ & $2.1 \pm 0.5$ & 0.04 & 0.840 & $1.9 \pm 0.4$ & $2.2 \pm 0.3$ & 4.51 & 0.043 \\
\hline Behavior control & $2.1 \pm 0.5$ & $2.2 \pm 0.4$ & 0.09 & 0.769 & $1.9 \pm 0.4$ & $2.3 \pm 0.4$ & 3.64 & 0.067 \\
\hline General functioning & $1.7 \pm 0.4$ & $1.9 \pm 0.5$ & 0.01 & 0.934 & $1.5 \pm 0.4$ & $2 \pm 0.3$ & 11.75 & 0.002 \\
\hline
\end{tabular}

FAD, Family Assessment Device. 
Table 3 Emotional/behavioral problems of the patient and control groups

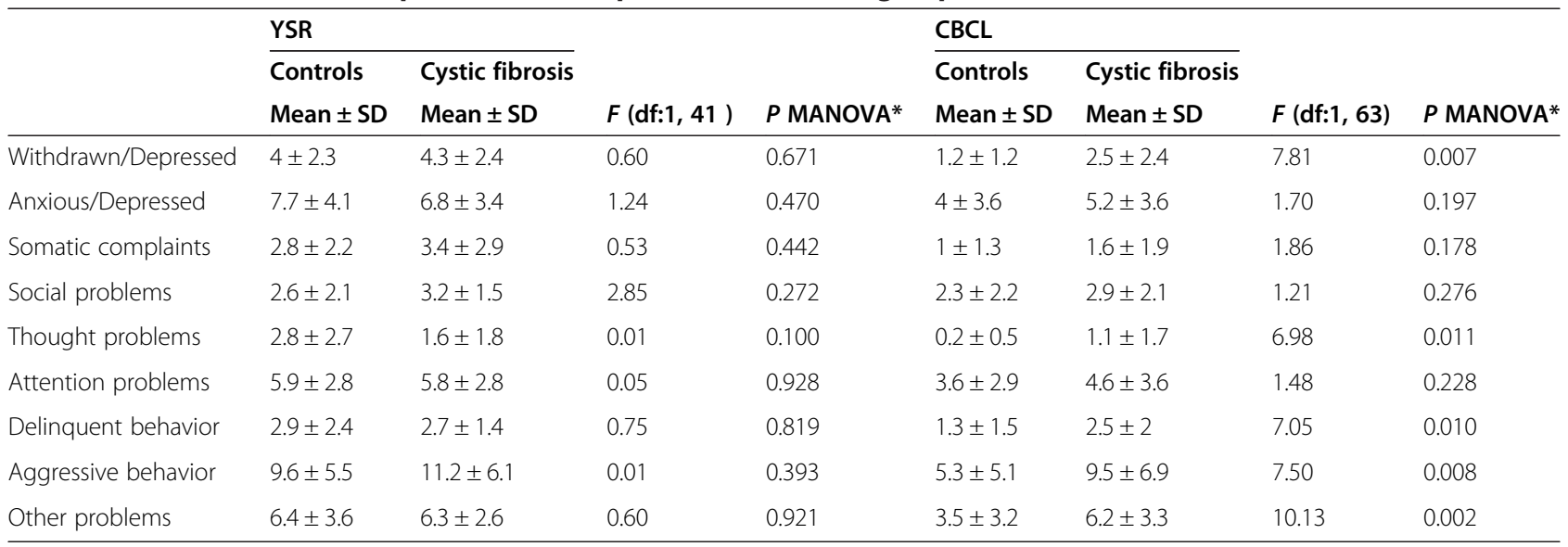

YSR, Youth Self-Report; CBCL, Child Behavior Checklist.

*sex and age were used as covariates.

illness perform lower levels of self-esteem than children with chronic illness [42], and so have similar studies focusing on adolescents with CF [44]. Issues related to small stature [18], delayed puberty [45], and deterioration of the symptoms [41] become more intense during adolescence for the young people with $\mathrm{CF}$ and may probably have a strong impact on self-esteem. In our study though, this fact could not be examined because of the restricted age range of the measure used (up to 13 years).

Children and adolescents with CF appear to have more difficulties in social adjustment when compared to their healthy peers. There is evidence that the presence of a childhood disability is associated with elevated use of health care services and the youth are four times as likely to be hospitalized and have eight times as many total hospital days as the general population [15]. More specifically, repeated absences and a lack of participation in recreational and sport activities, secondary to poor health, place children with $\mathrm{CF}$ at risk of social isolation due to diminished contacts and impaired leisure activities. Moreover, patients with CF must often be absent from school, resulting in delayed school performance and a sense of alienation [38].

Comparisons of the reports of children about their emotional/ behavioral problems showed no significant differences between the patients and controls in the present study. However, the parents of children with $\mathrm{CF}$ reported more emotional and behavioral difficulties in their offspring than did the parents of healthy children. Discrepancies between parents and children as informants have been reported before in studies of child psychopathology $[6,26]$. A possible interpretation for the discrepancy between the parents' and children's reports may relate to the fact that the parents of medically ill children are likely to be quite distressed, and as such may overestimate the externalizing behaviors (i.e., disobedience or other behavior problems) of their children or may underestimate their children's ability to adapt to illness [46]. On the other hand, medically ill children may minimize and underreport their distress or symptoms as part of their adaptation to illness [47].

The present findings should be interpreted in the context of some limitations. First, the sample size was rather small, leading to a low statistical power. Second, patients with moderate or severe pulmonary impairment were under-represented in this sample. Therefore, this study did not manage to examine the relation of the medical background of the patients (e.g. pulmonary impairment, past history, or severity of respiratory infections) to dysfunction of social adjustment or other psychosocial outcomes. Third, the study did not examine the effect of socioeconomic status on the reported differences, threatening generalizability of the results. Lastly, the cross-sectional design of the study did not allow us to examine variations over time.

\section{Conclusions}

The observed elevated levels of emotional/ behavioral problems and the difficulties in social adjustment of children and adolescents with CF indicate the need for the establishment of a collaborative treatment approach among medical and psychosocial personnel. Discrepancies between parents and children recommend the use of different sources of information. Future research should focus on the analysis of the variables that may mediate the effect on the psychosocial functioning of young patients and their families. Further investigation and analysis will be important to examine the relationship between advancing age, disease burden, and unfavorable psychosocial impact, as well as the different perceptions of children and their parents. 
Families with children who suffer from CF make great effort to provide the best possible disease management, hoping to insure their children's best quality and extension of life. A biopsychosocial approach should emphasize the assessment and treatment of the psychosocial distress of these patients alongside multiple somatic treatments.

\section{Competing interests}

The authors declare that they have no competing interests.

\section{Authors' contributions}

KK conceptualized and carried out the study and drafted the manuscript. GG conceptualized and prepared the manuscript. SDi and SDo participated in the study design and patient screening and enrollment. SC and CB participated in the study design and drafted the manuscript. GK conceptualized and coordinated the study. All authors read and approved the final manuscript.

\section{Author details}

'Department of Child Psychiatry, Athens University Medical School, Aghia Sophia Children's Hospital, Athens, Greece. ${ }^{2}$ Cystic Fibrosis Unit, Aghia Sophia Children's Hospital, Athens, Greece. ${ }^{3}$ Athens University Medical School, Athens, Greece.

Received: 10 November 2013 Accepted: 3 June 2014

Published: 11 June 2014

\section{References}

1. Katon WJ: Epidemiology and treatment of depression in patients with chronic medical illness. Dialogues Clin Neurosci 2011, 13:7-23.

2. Lewis M, Vitulano LA: Biopsychosocial issues and risk factors in the family when the child has a chronic illness. Child Adolesc Psychiatr Clin N Am 2003, 12:389-399. v.

3. Apter A, Farbstein I, Yaniv I: Psychiatric aspects of pediatric cancer. Child Adolesc Psychiatr Clin N Am 2003, 12:473-492. vii.

4. von Leupoldt A, Kenn K: The psychology of chronic obstructive pulmonary disease. Curr Opin Psychiatry 2013, 26:458-463.

5. Glazebrook C, Hollis C, Heussler H, Goodman R, Coates L: Detecting emotional and behavioral problems in paediatric clinics. Child Care Health Dev 2003, 29:141-149.

6. Pinquart $M$, Shen $Y$ : Behavior problems in children and adolescents with chronic physical illness: a meta-analysis. J Pediatr Psychol 2011, 36:1003-1016

7. Pinquart $M$, Shen $Y$ : Depressive symptoms in children and adolescents with chronic physical illness: an updated meta-analysis. J Pediatr Psychol 2011, 36:375-384

8. Knapp PK, Harris ES: Consultation-liaison in child psychiatry: a review of the past 10 years. Part I: Clinical findings. J Am Acad Child AdolesC Psychiatry 1998, 37:17-25.

9. Southern KW, Munck A, Pollitt R, Travert G, Zanolla L, Dankert-Roelse J, Castellani C, Group ECNSW: A survey of newborn screening for cystic fibrosis in Europe. J Cyst Fibros 2007, 6:57-65.

10. Salvatore D, Buzzetti $R$, Baldo E, Forneris MP, Lucidi V, Manunza D, Marinelli I, Messore B, Neri AS, Raia V, Furnari ML, Mastella G: An overview of international literature from cystic fibrosis registries. Part 3. Disease incidence, genotype/phenotype correlation, microbiology, pregnancy, clinical complications, lung transplantation, and miscellanea. J Cyst Fibros 2011, 10:71-85.

11. Farrell PM: The prevalence of cystic fibrosis in the European Union. J Cyst Fibros 2008, 7:450-453.

12. Mehta G, Macek M Jr, Mehta A: Cystic fibrosis across Europe: EuroCareCF analysis of demographic data from 35 countries. J Cyst Fibros 2010, 9(Suppl 2):S5-s21.

13. Ratjen F, Doring G: Cystic fibrosis. Lancet 2003, 361:681-689.

14. Boucher RC: Cystic fibrosis. In Harrison's principles of internal medicine. 17th edition. Edited by Fauci AS, Braunwal E, Kasper DL, Hauser SL, Longo DL, Jameson JL, Loscalzo J. New York: Mc Graw-Hill Medical; 2008:1632-1635.

15. Turkel S, Pao M: Late consequences of chronic pediatric illness. Psychiatr Clin North Am 2007, 30:819-835.

16. Gledhill J, Rangel L, Garralda E: Surviving chronic physical illness: psychosocial outcome in adult life. Arch Dis Child 2000, 83:104-110.
17. Reis HT, Collins WA, Berscheid E: The relationship context of human behavior and development. Psychol Bull 2000, 126:844-872.

18. Davis PB, Kercsmar CM: Growth in children with chronic lung disease. N Engl J Med 2000, 342:887-888.

19. Szyndler JE, Towns SJ, van Asperen PP, McKay KO: Psychological and family functioning and quality of life in adolescents with cystic fibrosis. J Cyst Fibros 2005, 4:135-144.

20. Bywater EM: Adolescents with cystic fibrosis: psychosocial adjustment. Arch Dis Child 1981, 56:538-543.

21. Drotar D, Doershuk CF, Stern RC, Boat TF, Boyer W, Matthews L: Psychosocial functioning of children with cystic fibrosis. Pediatrics 1981, 67:338-343.

22. Bregnballe V, Thastum M, Schiøtz PO: Psychosocial problems in children with cystic fibrosis. Acta Paediatr 2007, 96:58-61.

23. Steinhausen HC, Schindler HP: Psychosocial adaptation in children and adolescents with cystic fibrosis. J Dev Behav Pediatr 1981, 2:74-77.

24. Thompson RJ, Gustafson KE, Gil KM, Godfrey J, Murphy LM: Illness specific patterns of psychological adjustment and cognitive adaptational processes in children with cystic fibrosis and sickle cell disease. J Clin Psychol 1998, 54:121-128.

25. Cruz I, Marciel KK, Quittner AL, Schechter MS: Anxiety and depression in cystic fibrosis. Semin Respir Crit Care Med 2009, 30:569-578.

26. Bennett DS: Depression among children with chronic medical problems: a meta-analysis. J Pediatr Psychol 1994, 19:149-169.

27. Battle J: Culture-free self-esteem inventory for children and adults. Seattle, WA: Special Child Publications; 1981.

28. Weissman MM, Bothwell S: Assessment of social adjustment by patient self-report. Arch Gen Psychiatry 1976, 33:1111-1115.

29. Mufson L, Dorta KP, Wickramaratne $P$, Nomura $Y$, Olfson M, Weissman MM: A randomized effectiveness trial of interpersonal psychotherapy for depressed adolescents. Arch Gen Psychiatry 2004, 61:577-584.

30. Clemente C, Tsiantis J, Kolvin I, Ba G, Christogiorgos S, Lee C, Taylor B, Miller R: Social adjustment in three cultures: data from families affected by chronic blood disorders. A sibling studys. Haemophilia 2003, 9:317-324.

31. Epstein NB, Baldwin LM, Bishop DS: The mcmaster family assessment device. J Marital Fam Ther 1983, 9:171-180.

32. Achenbach TM: Manual for the youth self report and 1991 profile. Burlington, VT, USA: University of Vermont, Department of Psychiatry; 1991.

33. Le Blanc LA, Goldsmith T, Patel DR: Behaviorial aspects of chronic illness in chilsren and adolescents. Pediatr Clin Am 2003, 50:859-878.

34. Herzer M, Godiwala N, Hommel KA, Driscoll K, Mitchell M: Family functioning in the context of pediatric chornic conditrions. J Dev Behav Pediatr 2010, 31:26.

35. levers CE, Drotar D: Family and parental functioning in cystic fibrosis. J Dev Behav Pediatr 1996, 17:48-55.

36. Smetana JG, Campione-Barr N, Metzger A: Adolescent development in interpersonal and societal contexts. Annu Dev Psychol 2006, 57:255-284.

37. Santorck JW: Adolescence. 8th edition. Boston: Mc Graw-Hill; 2000.

38. Yeo M, Sawyer S: Chronic illness and disability. Br Med J 2005, 330:721-723.

39. Eiser C, Berrenberg J: Assessing the impact of chronic disease on the relationship between parent and their adolescents. J Psychosom Res 1995, 39:109-114

40. Hegarty M, Macdonald J, Watter P, Wilson C: Quality of life in young people with cystic fibrosis: effects of hospitalization, age and gender and differences in parent/child perceptions. Child Care Health Dev 2009, 35:462-468

41. Pinquart M: Self-esteem of children and adolescents with chronic illness: a meta-analysis. Child Care Health Dev 2013, 39:153-161.

42. Cowen L, Mok J, Corev M, Mac Millan H, Simmons R, Levison H: Psychological adjustment of the family with a member who has cystic fibrosis. Pediatrics 1986, 77:745-753.

43. Lewis BL, Khaw KT: Family Functioning as a mediating variable affecting psychosocial adjustment of children with cystic fibrosis. J Pediatr 1982 101:636-640.

44. Sawyer SM, Rosier MJ, Phelan PD, Bowes G: The self-image of adolescents with cystic fibrosis. J Adolesc Health 1995, 16:204-208.

45. Arrigo T, Rulli I, Sferlazzas C, De Luca F: Pubertal development in cystic fibrosis: an overview. J Pediatr Endocrinol Metab 2003, 2:267-270. 
46. Canning EH, Canning RD, Boyce WT: Depressive symptoms and adaptive style in children with cancer. J Am Acad Child Adolesc Psychiatry 1992, 31:1120-1124.

47. Canning EH, Hanser SB, Shade KA, Boyce WT: Maternal distress and discrepancy in reports of psychopathology in chronically ill children. Psychosomatics 1993, 34:506-511.

doi:10.1186/1751-0759-8-13

Cite this article as: Kostakou et al:: Psychosocial distress and functioning of Greek youth with cystic fibrosis: a cross-sectional study. BioPsychoSocial Medicine 2014 8:13.

\section{Submit your next manuscript to BioMed Central and take full advantage of:}

- Convenient online submission

- Thorough peer review

- No space constraints or color figure charges

- Immediate publication on acceptance

- Inclusion in PubMed, CAS, Scopus and Google Scholar

- Research which is freely available for redistribution 